Commons Select Committee meeting FOE listed a series of developmental setbacks that provokes sober reflection. The Enrico-Fermi FBR in Detroit, Michigan, for example, was constantly beset by potentially dangerous disruptions during its sporadic, nine-year operational life, while more recently the Soviet BN-350 FBR on the Caspian coast has suffered several serious leaks in the steam generating plant. Welding has, indeed, proved a persistent problem, and has delayed the full-power operation of the Dounreay PFR. The molten sodium used in the cooling circuits is highly corrosive. Void formation-the occurrence of gas bubbles in the coolant-is another major problem common to all FBRs and has still to be overcome.

Remaining hitches in reactor technology can, it is argued, be straightened out during the 20-year run-up to a commercial FBR network. But doubts linger over the associated fuel cycle. They arise not only at the technological level, where experience already gained by British Nuclear Fuels Ltd has

\title{
How the FBR is different
}

NUCLEAR power installations harness the heat energy released during controlled nuclear fission, and convert it into usable electricity. Fast breeder reactors (FBRs) differ from existing commercial reactors (known as "thermal" reactors) because, along with energy, they produce more fissile material than they consume-a self-sustaining bonus which could considerably stretch world uranium resources.

Like fast breeders, thermal reactors produce some plutonium but only in minute amounts. This is because more than $97 \%$ of the uranium used in thermal reactors fuel is non-fissile uranium238 , which is partially converted into plutonium while fission of the small fraction of uranium-235 proceeds. But plutonium is itself fissile, and most of it rapidly breaks down again, adding to the overall energy output of the system. (The Canadian CANDU reactor is designed to take full benefit of this phenomenon, optimising fuel efficiency.) Thus only about $1 \%$ of spent fuel from thermal reactors remains as plutonium.

On the other hand, the FBR can breed plutonium, using fast neutrons rather than the slow neutrons used in thermal reactors. Although fast neutrons are less efficient in inducing fission, the chain reaction they set up produces the greater number of additional neutrons needed to convert uranium-238. Consequently, the moderators used to decclerate fast neutrons in thermal reactors are absent from fast breeders.

The problem of decreased fission efficiency is overcome by pushing the neutron density in FBR fuel elements above the level achieved in thermal reactors. This means that the core assembly must be extremely compact, while the concentration of fissile material must be boosted way above the maximum enrichment factor of $3 \%$ needed in thermal fuel.

The $14 \mathrm{MW}$ experimental FBR at Dounreay (DFR), for instance, uses $75^{\prime \prime}$. enriched metallic uranium in a core of little more than 100 litres. At Dounreay. as in all FBRs, the uranium-238 needed for breeding is located in a "blanket" around the core, where the neutron density is no longer sufficient to break down the fissile plutonium formed.

In assessing the doubling time of an FBR - the period required for the reactor to reproduce the amount of fissile material originally in the reactor corethe entire fuel cycle is considered. At any onc instant, for example, fissile matcrial may be within the reactor itself, undergoing cooling, reprocessing or refabrication, or in transport.
It is thus possible to control the doubling time by retiming any of the operations involved in the cycle. Current UKAEA plans envisage a doubling time of about 28 years for Britain's first commercial FBRs early next century, a figure that neatly matches the expected expansion rate of the reactors themselves.

With the high neutron density in an FBR core, heat is generated more efficiently than in thermal reactors. Thus, while most thermal reactors operate at temperatures well below $300^{\circ} \mathrm{C}$, the $250 \mathrm{MW}$ prototype FBR at Dounreay (PFR) has a design outlet temperature of almost $600^{\circ} \mathrm{C}$. Fast breeder fuel elements must be designed to withstand the extra heat.

It soon became apparent that the metallic uranium core used in the DFR would melt at operating temperatures above $350{ }^{\circ} \mathrm{C}$. As a result, the PFR is fuelled by sintered pellets of uranium oxide and plutonium oxide. Uranium-235 is not an essential component, however, and fast breeders of the future could be fuelled with fissile plutonium-239 alone, though uranium-238 breeding material would, of course, have to be present. It is in by-passing the need for the scarce uranium-235 isotope that the FBR can stretch available uranium resources.

The fast breeders so far developed in Britain, France, Germany, Japan, the USA and the USSR are all designed to use liquid metal coolant circuits to carry the heat away from the core. They are therefore known as liquid metal fast breeder reactors. By using sodium, which is normally molten between $98^{\circ} \mathrm{C}$ and $880^{\circ} \mathrm{C}$, the unnecessary hazards associated with pressurised operation procedures can be avoided.

To minimise the risk of radioactive contamination, sodium circulated around the core in the primary reactor chamber passes its collected heat onto a secondary, external sodium circuit shielded from radiation. This circuit in turn carries heat to the steam generators.

FBR development has not run altogether smoothly. After 17 years operational experience with the DFR and the $P F R$, the UK nuclear industry is still struggling with problems in the steam generating plant of the PFR. The CEGB, however, which is prepared to have $20 \mathrm{GW}$ of FBR capacity plugged into the national grid by the year 2000 , has also declared a watchful interest in the development of helium-cooled fast breeders, which could provide an even better alternative to the liquid metal FBRs. been encouraging, if somewhat controversial, but also at environmental and political levels.

In a submission last year to the Royal Commission on Environmental Pollution (RCEP), which is due to publish a report on radiological hazards before Mr Benn's autumn decision, the UKAEA covered the environmental issues. Its arguments that the hazards of long-lived, highly active wastes can be satisfactorily controlled are likely to tip the scales in favour of the FBR. $\mathrm{Mr}$ Benn has indicated that the RCEP report will influence his decision, and it is now clear that the Commission itself, for some time reportedly divided over nuclear issues, will back CFR-1. In a recent reference to commercial FBR development Sir Brian Flowers, Chairman of the RCEP, said that while the implications and alternatives had not been explored with the level of resources and dedication devoted to the nuclear programme itself, he would not oppose the building of CFR-1 alone.

But the social and political implications of FBR reprocessing most worry those who believe that one step forward to CFR-1 will carry too much momentum for subsequent withdrawal. Though FBRs produce plutonium at a slower net rate than thermal reactors their proliferation would introduce the need for widespread plutonium transport. On UKAEA reckoning there could be 250 tons at various stages of the fuel cycle in Britain by the year 2000.

FBR opponents fear that the security measures required would be not far short of Draconian. A Bill to introduce the arming of nuclear security police has already been passed by the UK Parliament, and a US Government official this month called for an armed international security force. Additional doubts have been expressed about the sort of political arrangements needed to ensure worldwide adherence to safeguards such as those already implemented on a more limited scale by the International Atomic Energy Agency.

In France, the political and technological fears have led to civil disturbances involving qualified and wellinformed opponents of the 1,200 MW Superphénix project now beginning on on the Rhône. But in Britain there is little public awareness of the issues. Sir Brian Flowers recently charged that the UK Government apparently preferred to keep things in the dark. Similar concern has also led the Liberal Peer, Lord Avebury, to call for a Green Paper once the RCEP report is available, and to hint that a national referendum would not be inappropriate. Mr Benn has said only that a questionnaire will be produced for limited, and so far unspecified, circulation.

Though it is clear that the case for 